\title{
Fixed-order Control of LTI Systems Subject to Polytopic Uncertainty via the Concept of Strictly Positive Realness
}

\author{
Mahdieh S. Sadabadi and Alireza Karimi
}

\begin{abstract}
This paper deals with the problem of fixed-order controller design of LTI continuous-time and discrete-time polytopic systems via homogeneous polynomially parameterdependent Lyapunov matrices. The proposed method is based on the concept of Strictly Positive Realness (SPRness) of a transfer function depending on a parameter-dependent gain. To convert the problem to a set of LMI conditions, the parameterdependent gain is determined $a$ priori by means of a parameterdependent state feedback controller. Simulation results and a comparison with recent existing methods demonstrate the effectiveness of the proposed approach.
\end{abstract}

\section{INTRODUCTION}

The problem of fixed-order controller design is a theoretically challenging issue in control theory and it has attracted remarkable attention due to its great importance in practice. The non-convexity of the set of all fixed-order stabilizing controllers for a given plant is the main source of difficulty in solving such problem [1]. To address the problem, various approaches have been developed, e.g. nonsmooth non-convex-based [2]-[4] and LMI-based methods [5]-[7].

The problem of fixed-order controller design becomes more challenging in case of uncertainties in the plant model due to parameter drifting, unmodeled dynamics, modeling errors, etc. In this case, the main objective is to design a fixed-order controller which guarantees the robust stability as well as the robust performance of the uncertain system. To solve the problem, several LMI-based methods have emerged in the literature, e.g. the methods of [8]-[11] in polynomial framework and the methods of [12]-[22] in state space framework. In the state space approaches, some slack variables are used as a tool to decouple the product of closedloop matrices and Lyapunov matrices leading to a sequence of sufficient LMI conditions.

Recently, several slack variable-based approaches to fixedorder controller design of polytopic systems, which rely on the concept of Strictly Positive Realness (SPRness) of transfer functions, have been developed, e.g. [8]-[11], [20][22]. Moreover, it can be shown that most of the existing slack variable-based methods, e.g. [6], [12]-[19], implicitly rely on the concept of SPRness [23]. The main idea behind these approaches is to fix the slack variables a priori using some methods, e.g. initial output feedback controllers or a desired closed-loop characteristics polynomial. Therefore,

This research work is financially supported by the Swiss National Science Foundation under Grant No. 200020-130528.

M. S. Sadabadi and A. Karimi are with the Automatic Control Laboratory, Ecole Polytechnique Fédérale de Lausanne (EPFL), Lausanne, Switzerland.

Corresponding author: Alireza Karimi (alireza.karimi@epfl.ch) the SPRness of the transfer functions is parameterized by some LMIs thanks to KYP Lemma [24]. The way the slack variables are fixed is very crucial and affects the performance as well as the conservatism of the fixed-order control approach.

In this paper, necessary and sufficient conditions for fixed-order controller design of both continuous-time and discrete-time systems by means of homogeneous polynomially parameter-dependent Lyapunov matrices are presented. The focus of this paper is on systems subject to polytopic uncertainty. The proposed approach relies on the concept of SPRness of a special transfer function in which the slack matrices are determined a priori using an initial parameterdependent state feedback controller. Continuous-time and discrete-time fixed-order controller synthesis is treated in a unified manner. Furthermore, it is theoretically and numerically demonstrated that the proposed approach allows fixedorder stabilizing $\left(H_{\infty}\right)$ controller synthesis which potentially use less decision variables than some existing approaches, e.g. [18].

The organization of the paper is as follows. The problem formulation and preliminaries are given in Section II. Section III proposes the main results of the problem of fixed-order $H_{\infty}$ controller design of LTI continuous-time and discretetime systems with polytopic uncertainty. Simulation results are presented in Section IV. Finally, Section V concludes the paper.

Throughout the paper, matrices $I$ and 0 are the identity matrix and the zero matrix of appropriate dimensions, respectively. The symbol $T$ and $\star$ are used to show the matrix transpose and the symmetric blocks, respectively. For symmetric matrices, $P>0(P<0)$ indicates the positivedefiniteness (the negative-definiteness).

\section{Problem Formulation AND Preliminaries}

Consider a linear time-invariant (LTI) system described by the following dynamical equations:

$$
\begin{aligned}
\delta\left[x_{g}(t)\right] & =A_{g} x_{g}(t)+B_{g} u(t)+B_{w} w(t) \\
z(t) & =C_{z} x_{g}(t)+D_{z u} u(t) \\
y(t) & =C_{g} x_{g}(t)
\end{aligned}
$$

where $x_{g} \in \mathbb{R}^{n}, u \in \mathbb{R}^{n_{i}}, w \in \mathbb{R}^{r}, y \in \mathbb{R}^{n_{o}}$, and $z \in$ $\mathbb{R}^{s}$ are the state, the control input, the exogenous input, the measured output, and the controlled output, respectively. The symbol $\delta[\cdot]$ presents the derivative term for continuous-time $(\delta[x(t)]=d x / d t)$ and the forward operator for discrete-time systems $(\delta[x(t)]=x(t+1))$. Matrices $A_{g}, B_{g}, B_{w}, C_{z}, C_{g}$, 
and $D_{z u}$ belong to the following uncertainty domain:

$$
\begin{aligned}
\Omega & =\left\{\left(A_{g}(\lambda), B_{g}(\lambda), B_{w}(\lambda), C_{z}(\lambda), C_{g}(\lambda), D_{z u}(\lambda)\right)\right. \\
& \left.=\sum_{i=1}^{q} \lambda_{i}\left(A_{g_{i}}, B_{g_{i}}, B_{w_{i}}, C_{z_{i}}, C_{g_{i}}, D_{z u_{i}}\right)\right\}
\end{aligned}
$$

where $\lambda=\left[\lambda_{1}, \ldots, \lambda_{q}\right]$ belongs to the following unit simplex:

$$
\Lambda_{q}=\left\{\lambda_{1}, \ldots, \lambda_{q} \mid \sum_{i=1}^{q} \lambda_{i}=1, \quad \lambda_{i} \geq 0\right\}
$$

and matrices $A_{g_{i}}, B_{g_{i}}, B_{w_{i}}, C_{z_{i}}, C_{g_{i}}$, and $D_{z u_{i}}$ are the $i$-th vertex of the polytope. The main objective of this paper is to design a robust fixed-order stabilizing $\left(H_{\infty}\right)$ controller for the polytopic system given by:

$$
\begin{aligned}
\delta\left[x_{c}(t)\right] & =A_{c} x_{c}(t)+B_{c} y(t) \\
u(t) & =C_{c} x_{c}(t)+D_{c} y(t)
\end{aligned}
$$

where $A_{c} \in \mathbb{R}^{m \times m}$ and $B_{c}, C_{c}$, and $D_{c}$ are of appropriate dimensions.

The problem of dynamic output-feedback controller synthesis can be equivalently transformed to a static output feedback one by introducing an augmented plant as follows [25]:

$$
\begin{aligned}
\delta\left[\bar{x}_{g}(t)\right] & =\bar{A}_{g}(\lambda) \bar{x}_{g}(t)+\bar{B}_{g}(\lambda) u(t)+\bar{B}_{w}(\lambda) w(t) \\
z(t) & =\bar{C}_{z}(\lambda) \bar{x}_{g}(t)+\bar{D}_{z u}(\lambda) u(t) \\
y(t) & =\bar{C}_{g}(\lambda) \bar{x}_{g}(t)
\end{aligned}
$$

where

$$
\begin{gathered}
\bar{A}_{g}(\lambda)=\left[\begin{array}{cc}
A_{g}(\lambda) & 0 \\
0 & 0_{m}
\end{array}\right], \quad \bar{B}_{g}(\lambda)=\left[\begin{array}{cc}
0 & B_{g}(\lambda) \\
I_{m} & 0
\end{array}\right] \\
\bar{B}_{w}(\lambda)=\left[\begin{array}{c}
B_{w}(\lambda) \\
0
\end{array}\right], \quad \bar{C}_{g}(\lambda)=\left[\begin{array}{cc}
0 & I_{m} \\
C_{g}(\lambda) & 0
\end{array}\right] \\
\bar{C}_{z}(\lambda)=\left[\begin{array}{ll}
C_{z}(\lambda) & 0
\end{array}\right], \quad \bar{D}_{z u}(\lambda)=\left[\begin{array}{ll}
0 & D_{z u}(\lambda)
\end{array}\right]
\end{gathered}
$$

Closed-loop system $H_{z w}(\lambda)$, transfer function from $w$ to $z$, has the following state space realization:

$$
\begin{aligned}
\delta[x(t)] & =A(\lambda) x(t)+B(\lambda) w(t) \\
z(t) & =C(\lambda) x(t)
\end{aligned}
$$

where $x=\bar{x}_{g}$ and

$$
\begin{gathered}
K=\left[\begin{array}{ll}
A_{c} & B_{c} \\
C_{c} & D_{c}
\end{array}\right] \\
A(\lambda)=\bar{A}_{g}(\lambda)+\bar{B}_{g}(\lambda) K \bar{C}_{g}(\lambda) \\
B(\lambda)=\bar{B}_{w}(\lambda) \\
C(\lambda)=\bar{C}_{z}(\lambda)+\bar{D}_{z u}(\lambda) K \bar{C}_{g}(\lambda)
\end{gathered}
$$

The remains of this section provide basic lemmas which will be used throughout this paper.

Lemma 1: (Kalman-Yakubovich-Popov (KYP) Lemma [24]) A square transfer function $H=\left[\begin{array}{l|l}A & B \\ \hline C & D\end{array}\right]$ is SPR if and only if there exists a Lyapunov matrix $P>0$ such that

For continuous-time systems:

$$
\left[\begin{array}{cc}
A^{T} P+P A & P B-C^{T} \\
B^{T} P-C & -D-D^{T}
\end{array}\right]<0
$$

For discrete-time systems:

$$
\left[\begin{array}{cc}
A^{T} P A-P & A^{T} P B-C^{T} \\
B^{T} P A-C & B^{T} P B-D-D^{T}
\end{array}\right]<0
$$

Lemma 2: The following statements are equivalent [20], [21]:

1) A square transfer function $H=\left[\begin{array}{l|l}A & B \\ \hline C & D\end{array}\right]$ is $\mathrm{SPR}$ with Lyapunov matrix $P>0$.

2) $H^{-1}=\left[\begin{array}{c|c}A-B D^{-1} C & -B D^{-1} \\ \hline D^{-1} C & D^{-1}\end{array}\right]$ is SPR with Lyapunov matrix $P>0$.

As a result, the following inequalities are equivalent: Continuous-time systems:

$$
\begin{aligned}
& {\left[\begin{array}{cc}
A^{T} P+P A & \star \\
B^{T} P-C & -D-D^{T}
\end{array}\right]<0} \\
& {\left[\begin{array}{cc}
A^{* T} P+P A^{*} & \star \\
-D^{-T} B^{T} P-D^{-1} C & -D^{-1}-D^{-T}
\end{array}\right]<0}
\end{aligned}
$$

Discrete-time systems:

$$
\begin{aligned}
& {\left[\begin{array}{cc}
A^{T} P A-P & \star \\
B^{T} P A-C & B^{T} P B-D-D^{T}
\end{array}\right]<0} \\
& {\left[\begin{array}{cc}
A^{* T} P A^{*}-P & \star \\
-\left(B D^{-1}\right)^{T} P A^{*}-D^{-1} C & \left(B D^{-1}\right)^{T} P B D^{-1}- \\
& \left(D^{-1}+D^{-T}\right)
\end{array}\right]<0}
\end{aligned}
$$

where $A^{*}=A-B D^{-1} C$.

\section{MAIN RESULTS}

The following theorems present necessary and sufficient conditions for stabilizing $\left(H_{\infty}\right)$ static output feedback controller synthesis.

\section{A. Fixed-order Stabilizing Controller Design}

Theorem 1: The static output feedback controller $K=$ $X^{-1} L$ stabilizes the augmented polytopic system in (5) if and only if there exist a parameter-dependent gain $K_{s f}(\lambda)$, a Lyapunov matrix $P(\lambda)>0$, and two matrices $X$ and $L$ such that

For continuous-time systems:

$$
\left[\begin{array}{cc}
M^{T}(\lambda) P(\lambda)+P(\lambda) M(\lambda) & \star \\
\bar{B}_{g}^{T}(\lambda) P(\lambda)-N(\lambda) & -X-X^{T}
\end{array}\right]<0
$$

For discrete-time systems:

$$
\left[\begin{array}{cc}
M^{T}(\lambda) P(\lambda) M(\lambda)-P(\lambda) & \star \\
\bar{B}_{g}^{T}(\lambda) P(\lambda) M(\lambda)-N(\lambda) & \left(\bar{B}_{g}^{T}(\lambda) P(\lambda) \bar{B}_{g}(\lambda)\right. \\
& \left.-X-X^{T}\right)
\end{array}\right]<0
$$

where

\section{Proof:}

$$
\begin{aligned}
M(\lambda) & =\bar{A}_{g}(\lambda)+\bar{B}_{g}(\lambda) K_{s f}(\lambda) \\
N(\lambda) & =X K_{s f}(\lambda)-L \bar{C}_{g}(\lambda)
\end{aligned}
$$

Sufficiency: According to KYP Lemma, the inequality $(13) /(14)$ indicates that the following transfer function is SPR with Lyapunov matrix $P(\lambda)$ :

$$
H=\left[\begin{array}{c|c}
M(\lambda) & \bar{B}_{g}(\lambda) \\
\hline X\left(K_{s f}(\lambda)-K C_{g}(\lambda)\right) & X
\end{array}\right]
$$


According to Lemma 2, the SPRness of $H$ implies that $H^{-1}$ with the following realization is also SPR with the same Lyapunov matrix $P(\lambda)$.

$$
H^{-1}=\left[\begin{array}{c|c}
A(\lambda) & -\bar{B}_{g}(\lambda) X^{-1} \\
\hline K_{s f}(\lambda)-K C_{g}(\lambda) & X^{-1}
\end{array}\right]
$$

The SPRness of $H^{-1}$ with $P(\lambda)$ implies that the closed-loop state matrix $A(\lambda)$ is stable with the parameter-dependent Lyapunov matrix $P(\lambda)$.

Necessity: Assume that $K$ stabilizes the closed loop polytopic system in (6)-(8). Let's choose $X=I\left(X+X^{T}>0\right)$, $L=K$, and $K_{s f}(\lambda)=K \bar{C}_{g}(\lambda)$. Then, the following transfer function matrix $H$ is SPR and (13)/(14) is satisfied.

$$
\begin{aligned}
H & =\left[\begin{array}{c|c}
M(\lambda) & \bar{B}_{g}(\lambda) \\
\hline 0 & I
\end{array}\right] \\
& =0 \times(x I-M(\lambda))^{-1} \times \bar{B}_{g}(\lambda)+I \\
& =I>0
\end{aligned}
$$

where $x=s$ and $x=z$ for continuous-time and discretetime case, respectively. Thus, this completes the proof.

\section{B. Relation between the Proposed Stabilizing Static Output Feedback Controller Design Method in [18] and Theorem 1}

In this subsection, we show that the proposed approach in [18] relies on the concept of SPRness of a transfer function where $A$-matrix is fixed by a parameter-dependent gain.

Lemma 3: Let $K_{s f}(\lambda)$ be a stabilizing parameterdependent gain for the continuous-time augmented system subject to polytopic uncertainty described by (5). Then, the following statements are equivalent:

(a) If there exist two matrices $X$ and $L$ such that the following transfer function is SPR:

$$
H(s)=\left[\begin{array}{c|c}
\bar{A}_{g}(\lambda)+\bar{B}_{g}(\lambda) K_{s f}(\lambda) & \bar{B}_{g}(\lambda) \\
\hline X K_{s f}(\lambda)-L C_{g}(\lambda) & X
\end{array}\right]
$$

(b) If there exist a Lyapunov matrix $P(\lambda)>0$, and two matrices $X$ and $L$ such that the following inequality holds:

$$
\left[\begin{array}{cc}
M^{T}(\lambda) P(\lambda)+P(\lambda) M(\lambda) & \star \\
\bar{B}_{g}^{T}(\lambda) P(\lambda)-\left(X K_{s f}(\lambda)-L \bar{C}_{g}(\lambda)\right) & -X-X^{T}
\end{array}\right]<0
$$

where $M(\lambda)=\bar{A}_{g}(\lambda)+\bar{B}_{g}(\lambda) K_{s f}(\lambda)$.

(c) If there are a Lyapunov matrix $P(\lambda)$ and matrices $F(\lambda), V(\lambda), X$, and $L$ such that (21) holds [18].

Then, $K=X^{-1} L$ is a robust static output feedback controller which stabilizes the augmented continuous-time system given in (5).

Proof: The statements $(a)$ and (b) directly results from KYP Lemma. Therefore, it is enough to show that (21) is equivalent to (20). Post-multiplying (21) by $Q(\lambda)$

$$
Q(\lambda)=\left[\begin{array}{cc}
I & 0 \\
\bar{A}_{g}+\bar{B}_{g} K_{s f} & \bar{B}_{g} \\
0 & I
\end{array}\right]
$$

and pre-multiplying by $Q^{T}(\lambda)$, the inequality given in (20) is obtained.
As a result, the parameter-dependent slack matrices $F(\lambda)$ and $V(\lambda)$ in (21) can be eliminated without conservatism. In fact, these matrices do not affect the final static output feedback controller; however, elimination of them leads to less computation time.

\section{Fixed-order $H_{\infty}$ Controller Design}

Theorem 2: (Continuous-time case) The $H_{\infty}$ static output feedback $K=X^{-1} L$ stabilizes the augmented polytopic system in (5) and ensures the robust performance $\left\|H_{z w}(\lambda)\right\|_{\infty}^{2}<\mu$, for all $\lambda \in \Lambda_{q}$, if and only if there exist a stabilizing parameter-dependent gain $K_{s f}(\lambda)$, a Lyapunov matrix $P(\lambda)>0$, and two auxiliary matrices $X$ and $L$ such that

$$
\left[\begin{array}{cccc}
M^{T}(\lambda) P(\lambda)+P(\lambda) M(\lambda) & \star & \star & \star \\
\bar{B}_{g}^{T}(\lambda) P(\lambda)-N(\lambda) & -X-X^{T} & \star & \star \\
B^{T}(\lambda) P(\lambda) & 0 & -\mu I & \star \\
\bar{C}_{z}(\lambda)+\bar{D}_{z u}(\lambda) K_{s f} & \bar{D}_{z u}(\lambda) & 0 & -I
\end{array}\right]<0
$$

where $M(\lambda)$ and $N(\lambda)$ are defined in (15).

Theorem 3: (Discrete-time case) The $H_{\infty}$ static output feedback $K=X^{-1} L$ stabilizes the augmented discrete-time polytopic system in (5) and guarantees $\left\|H_{z w}(\lambda)\right\|_{\infty}^{2}<\mu$, for all $\lambda \in \Lambda_{q}$, if and only if there exist a stabilizing parameterdependent gain $K_{s f}(\lambda)$, a Lyapunov matrix $P(\lambda)>0$, and two auxiliary matrices $X$ and $L$ such that (24) with $M(\lambda)$ and $N(\lambda)$ defined in (15) holds.

Remark: If the parameter-dependent gain $K_{s f}(\lambda)$ is given a priori and $P(\lambda)$ is considered as a homogeneous polynomial w.r.t. $\lambda$, the parameter dependent conditions in Theorem 1-3 can be handled by a sequence of LMI relaxations. Based on the results mentioned in [26], parameter-dependent LMIs with parameters in the unit simplex always have homogenous polynomially parameter-dependent solutions of sufficiently high degree. Moreover, they can be solved with no conservatism by a set of LMI relaxations. Therefore, we assume that the gain $K_{s f}(\lambda)$ is initialized by means of a parameter-dependent stabilizing $\left(H_{\infty}\right)$ state feedback controller.

In following, two new theorems for parameter-dependent $H_{\infty}$ state feedback controller design of both continuous-time and discrete-time augmented polytopic systems described by (5) are given.

\section{Parameter-dependent State Feedback Control Design}

A parameter-dependent $H_{\infty}$ state feedback controller $K_{s f}(\lambda)$ for the continuous-time and discrete-time augmented systems in (5) can be determined by the following theorems.

Theorem 4: (Continuous-time case) The parameterdependent state feedback controller $K_{s f}(\lambda)=Z(\lambda) F^{-1}(\lambda)$ stabilizes the augmented continuous-time polytopic systems in (5) and guarantees the desired performance $\left\|H_{z w}(\lambda)\right\|_{\infty}<\gamma$, for all $\lambda \in \Lambda_{q}$, if and only if there exist a parameter-dependent Lyapunov matrix $P(\lambda)>0$, matrices $F(\lambda)$, and $Z(\lambda)$ and a positive scalar $\delta>0$ such that (25) is satisfied.

Theorem 5: (Discrete-time case) The parameterdependent state feedback $K_{s f}(\lambda)=Z(\lambda) F^{-1}(\lambda)$ guarantees the stability and closed-loop performance $\left\|H_{z w}(\lambda)\right\|_{\infty}^{2}<\mu$ 


$$
\left[\begin{array}{ccc}
\left(\bar{A}_{g}(\lambda)+\bar{B}_{g}(\lambda) K_{s f}(\lambda)\right)^{T} F^{T}(\lambda)+F(\lambda)\left(\bar{A}_{g}(\lambda)+\bar{B}_{g}(\lambda) K_{s f}(\lambda)\right) & \star & \star \\
P(\lambda)-F^{T}(\lambda)+V(\lambda)\left(\bar{A}_{g}(\lambda)+\bar{B}_{g}(\lambda) K_{s f}(\lambda)\right) & -V(\lambda)-V^{T}(\lambda) & \star \\
\bar{B}_{g}^{T}(\lambda) F^{T}(\lambda)+L \bar{C}_{g}(\lambda)-X K_{s f}(\lambda) & \bar{B}_{g}^{T}(\lambda) V^{T}(\lambda) & -X-X^{T}
\end{array}\right]<0
$$

$$
\left[\begin{array}{cccc}
M^{T}(\lambda) P(\lambda) M(\lambda)-P(\lambda) & \star & \star & \star \\
\bar{B}_{g}^{T}(\lambda) P(\lambda) M(\lambda)-N(\lambda) & \bar{B}_{g}^{T}(\lambda) P(\lambda) \bar{B}_{g}(\lambda)-X-X^{T} & \star & \star \\
B^{T}(\lambda) P(\lambda) M(\lambda) & B^{T}(\lambda) P(\lambda) \bar{B}_{g}(\lambda) & -\mu I+B^{T}(\lambda) P(\lambda) B(\lambda) & \star \\
\bar{C}_{z}(\lambda)+\bar{D}_{z u}(\lambda) K_{s f}(\lambda) & \bar{D}_{z u}(\lambda) & 0 & -I
\end{array}\right]<0
$$

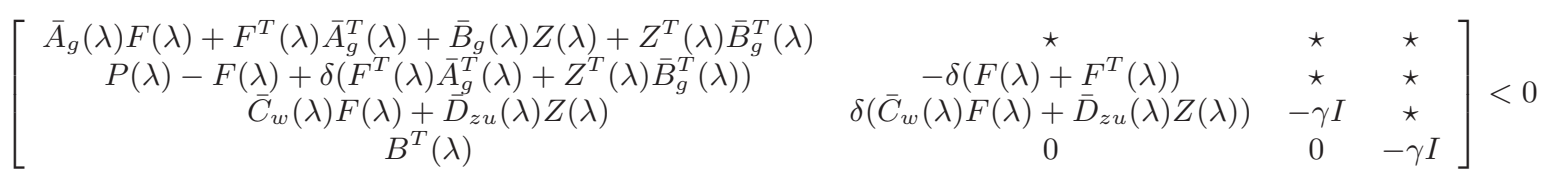

of the augmented discrete-time polytopic systems in (5), for all $\lambda \in \Lambda_{q}$, if and only if there exist parameter-dependent matrices $P(\lambda)>0, F(\lambda)$, and $Z(\lambda)$ such that (26) holds.

Remark: Theorem 4 and Theorem 5 can be used for the design of stabilizing parameter-dependent state feedback controllers for continuous-time and discrete-time systems by removing the third and forth rows and columns of matrices in (25) and (26), respectively.

\section{E. Controller Design Procedure}

The robust fixed-order $H_{\infty}$ controller design procedure includes the following steps:

Step 1: Choose the order of controller $(m)$ and construct the augmented system in (5).

Step 2: Set $j=1$ and design the parameter-dependent gain $K_{s f}^{[1]}(\lambda)$ for the augmented system using Theorem 4/Theorem 5.

Step 3: Choose the degree of the homogenous Lyapunov matrix $P(\lambda)$ and solve the convex optimization problem proposed in Theorem 2/Theorem 3 by constructing the LMI constraints in (23)/(24) (using e.g. ROLMIP [27]) to obtain the static output feedback controller $K^{[j]}$.

Step 4: If $\mu^{[j-1]}-\mu^{[j]}>\epsilon$, update the parameterdependent gain $K_{s f}(\lambda)$, i.e. $K_{s f}^{[j+1]}(\lambda)=K^{[j]} \bar{C}_{g}(\lambda)$, and go to Step 3 with $j \longleftarrow j+1$, else stop.

Theorem 6: The iterative algorithm leads to monotonic convergence of the upper bound on the $H_{\infty}$ norm.

Remark: It should be mentioned that the set of LMI constraints from parameter-dependent LMIs in Theorem 15 with parameters in the unit simplex are constructed using ROLMIP (Robust LMI Parser) [27]. ROLMIP is a computational MATLAB package which provides an interface for the users to construct a finite set of LMIs from parameterdependent LMIs with parameters in the unit simplex [27].

\section{Simulation Results}

In this section, several examples from the literature are given to evaluate the effectiveness of the proposed fixedorder control approach. A comparison with the recent existing methods is made. It should be noted that in all tables, the set $\left\{d_{Z}, d_{F}, d_{P_{s f}}, d_{P}\right\}$, respectively, denotes the degrees of the homogeneous polynomials $Z(\lambda), F(\lambda)$, and
TABLE I

MAXIMUM INTERVAL OF PARAMETER $a$ IN EXAMPLE 1

\begin{tabular}{|c|c|c|}
\hline Method & $\left\{d_{Z}, d_{F}, d_{P_{s f}}, d_{P}\right\}$ & $a$ \\
\hline Theorem 1 & $\{1,0,1,1\}$ & {$\left[\begin{array}{ll}-17.8 & 122.2\end{array}\right]$} \\
\hline$[19]$ & $\{-,-,-, 1\}$ & {$\left[\begin{array}{ll}3.6 & 82.2\end{array}\right]$} \\
\hline$[18]$ & $\{1,0,1,1\}$ & {$\left[\begin{array}{ll}-17.8 & 122.2\end{array}\right]$} \\
\hline$[30]$ & - & Non-applicable \\
\hline$[31]$ & - & Non-applicable \\
\hline
\end{tabular}

$P(\lambda)$ in Theorem 4 and 5 and the degree of homogeneous polynomially parameter-dependent Lyapunov matrix $P(\lambda)$ in Theorem 1-3 ${ }^{1}$ To solve the LMI problems in MATLAB, YALMIP [28] as the interface and SeDuMi [29]/MOSEK ${ }^{2}$ as the solver are used.

Example 1: Consider a third-order continuous-time polytopic system, borrowed from [19], with the following vertices:

$$
\begin{aligned}
A_{g_{1}} & =\left[\begin{array}{ccc}
-1 & 4 & 0 \\
0 & 0 & 1 \\
a & 6 & -1
\end{array}\right] ; \quad A_{g_{2}}=\left[\begin{array}{ccc}
-1 & 1 & 0 \\
0 & -5 & 1 \\
10 & 1 & -1
\end{array}\right] \\
B_{g_{1}} & =\left[\begin{array}{l}
0 \\
0 \\
1
\end{array}\right] ; \quad B_{g_{2}}=\left[\begin{array}{l}
0 \\
0 \\
1
\end{array}\right] \\
C_{g_{1}} & =\left[\begin{array}{lll}
1 & 1 & 0 \\
0 & 1 & 0
\end{array}\right] ; \quad C_{g_{2}}=\left[\begin{array}{lll}
1 & 1 & 0 \\
0 & 0 & 0
\end{array}\right]
\end{aligned}
$$

The main objective is to design a stabilizing static output feedback controller which leads to the largest interval of parameter $a$. Therefore, the system can be modeled with a polytope with three vertices, i.e. $\left.A_{g_{1}}\right|_{a=a_{\min }},\left.A_{g_{1}}\right|_{a=a_{\max }}$, and $A_{g_{2}}$. The minimum and maximum values of $a$ can be determined by a bisection algorithm. The results of Theorem 1 are compared with ones of [18], [19], [30], [31] in Table I. The proposed methods in Theorem 1 and [18] are both initialized with the same parameter-dependent state feedback $K_{s f}(\lambda)$.

As it is reported in [19], since matrix $C(\lambda)$ is not full row rank, the approaches of [30], [31] are not applicable. Results given in Table I indicate that the proposed method

\footnotetext{
${ }^{1}$ In this paper, the degree of the other slack variables of [18] is considered equal to one.

${ }^{2}$ Available online in http://www.mosek.com
} 


$$
\left[\begin{array}{cccc}
P(\lambda) & \star & \star & \star \\
F^{T}(\lambda) \bar{A}_{g}^{T}(\lambda)+Z^{T}(\lambda) \bar{B}_{g}^{T}(\lambda) & F(\lambda)+F^{T}(\lambda)-P(\lambda) & \star & \star \\
0 & \bar{C}_{w}(\lambda) F(\lambda)+\bar{D}_{z u}(\lambda) Z(\lambda) & I & \star \\
B^{T}(\lambda) & 0 & 0 & \mu I
\end{array}\right]>0
$$

TABLE II

UPPER BOUND OF $\left\|H_{z w}(\lambda)\right\|_{\infty}$ IN EXAMPLE 2

\begin{tabular}{|c|c|c|c|}
\hline Method & Iterations & $\gamma=\sqrt{\mu}$ & $\bar{K}$ \\
\hline [12] & 1 & 9.73 & \begin{tabular}{|ll}
0.56 & 5.08 \\
\end{tabular} \\
\hline [32] & 1 & 6.80 & {$[0.054 \quad 0.64]$} \\
\hline [19] & 1 & 2.33 & {$\left[\begin{array}{ll}0.45 & 4.19\end{array}\right]$} \\
\hline [20] & 5 & 1.79 & {$\left[\begin{array}{ll}77.16 & 608.87\end{array}\right]$} \\
\hline [33] & 30 & 1.66 & {$\left[\begin{array}{ll}130.35 & 939.37\end{array}\right]$} \\
\hline Theorem 2 & 5 & 1.78 & {$\left[\begin{array}{ll}9.36 & 69.57\end{array}\right]$} \\
\hline
\end{tabular}

in this paper and [18] lead to the best results among the others.

Example 2: Consider the following continuous-time polytopic system with two vertices [19]:

$$
\begin{aligned}
A_{g_{1}} & =\left[\begin{array}{ccc}
-0.9896 & 17.41 & 96.15 \\
0.2648 & -0.8512 & -11.39 \\
0 & 0 & -30
\end{array}\right] \\
A_{g_{2}} & =\left[\begin{array}{ccc}
-1.702 & 50.72 & 263.5 \\
0.2201 & -1.418 & -31.99 \\
0 & 0 & -30
\end{array}\right] \\
B_{g_{1}} & =\left[\begin{array}{c}
-97.78 \\
0 \\
30
\end{array}\right] ; B_{g_{2}}=\left[\begin{array}{c}
-85.09 \\
0 \\
30
\end{array}\right] ; B_{w}=\left[\begin{array}{l}
0 \\
1 \\
1
\end{array}\right] \\
C_{g} & =\left[\begin{array}{lll}
1 & 0 & 0 \\
0 & 1 & 0
\end{array}\right] ; C_{w}=\left[\begin{array}{lll}
1 & 0 & 0 \\
0 & 1 & 0 \\
0 & 0 & 1
\end{array}\right] ; D_{z u}=\left[\begin{array}{l}
0 \\
0 \\
0
\end{array}\right]
\end{aligned}
$$

The objective here is to design a static output feedback $H_{\infty}$ controller with linearly parameter-dependent Lyapunov matrices. To this end, an optimization problem, which is the minimization of the upper bound of $H_{\infty}$ norm $(\gamma)$ subject to a sequence of LMI constraints is solved. Resulting static output feedback initialized by parameter-dependent state feedback controller with $d_{Z}=1, d_{F}=0$, and $d_{P_{s f}}=2$ is given in Table II. The results are compared with the LMIbased methods in [12], [19], [20], [32] and the BMI-based method in [33]. For all cases, the degree of Lyapunov matrix $P$ is one.

Example 3: As the third example, consider the modified version of the pitch control of F4E, given in [34], described by the following state space matrices:

$$
\begin{aligned}
A_{g} & =\left[\begin{array}{cccc}
a_{11} & a_{12} & a_{13} & b_{1} \\
a_{21} & a_{22} & a_{23} & 0 \\
0 & 0 & -30 & 30 \\
0 & 0 & 0 & -10^{4}
\end{array}\right] ; \quad B_{g}=\left[\begin{array}{c}
0 \\
0 \\
0 \\
10^{4}
\end{array}\right] \\
C_{g} & =\left[\begin{array}{cccc}
c & 0 & 0 & 0 \\
0 & c & 0 & 0
\end{array}\right] ; \quad B_{w}=\left[\begin{array}{lll}
1 & 0 & 0 \\
0 & 1 & 0 \\
0 & 0 & 1 \\
0 & 0 & 0
\end{array}\right] \\
C_{w} & =\left[\begin{array}{llll}
1 & 0 & 0 & 0 \\
0 & 1 & 0 & 0 \\
0 & 0 & 1 & 0
\end{array}\right] ; \quad D_{z u}=\left[\begin{array}{l}
0 \\
0 \\
1
\end{array}\right]
\end{aligned}
$$

\begin{tabular}{|c|c|c|c|c|}
\hline Initialization & \multicolumn{2}{|c|}{ Theorem 4} & \multicolumn{2}{|c|}{ HIFOO } \\
\hline controller order & $m=0$ & $m=1$ & $m=0$ & $m=1$ \\
\hline degree of $P\left(d_{P}\right)$ & 1 & 1 & 1 & 2 \\
\hline Iterations & 4 & 2 & 30 & 30 \\
\hline$\gamma$ & 3.0780 & 3.1026 & 3.3378 & 2.2818 \\
\hline
\end{tabular}

TABLE III

PARAMETERS OF FOUR OPERATING POINTS IN EXAMPLE 3

\begin{tabular}{|c|c|c|c|c|}
\hline Operating points & 1 & 2 & 3 & 4 \\
\hline Mach number & 0.5 & 0.9 & 0.85 & 1.5 \\
\hline Altitude(ft) & 5000 & 35000 & 5000 & 35000 \\
\hline$a_{11}$ & -0.9896 & -0.6607 & -1.0702 & -0.5162 \\
$a_{12}$ & 17.41 & 18.11 & 50.72 & 29.96 \\
$a_{13}$ & 96.15 & 84.34 & 263.5 & 178.9 \\
$a_{21}$ & 0.2648 & 0.08201 & 0.2201 & -0.6896 \\
$a_{22}$ & -0.8512 & -0.6587 & -1.418 & -1.225 \\
$a_{23}$ & -11.39 & -10.81 & -31.99 & -30.38 \\
$b_{1}$ & -97.78 & -272.2 & -85.09 & -175.6 \\
\hline
\end{tabular}

TABLE IV

UPPER BOUND OF $\left\|H_{z w}(\lambda)\right\|_{\infty}$ IN EXAMPLE 3

where $0.5 \leq c \leq 1$ and parameters $a_{i j}, i=1,2 ; j=1,2,3$, and $b_{1}$ for four operating points are given in Table III.

The uncertainty of the system in (28) is in the form of a polytope with $q=8$ vertices. The proposed approach in [34] as well as the full-order controller design method of [35] are employed for the comparison purposes. It should be noted since the main assumption of [19] is that $C_{w}^{T} D_{z u}=0$, it cannot be applied to Example 3.

Theorem 2 is initialized by two different parameterdependent gain $K_{s f}(\lambda)$. In the first case, an initial parameterdependent state feedback controller is designed using Theorem 4 with $d_{Z}=0, d_{F}=0$, and $d_{P_{s f}}=1$. In the second case, $K_{s f}(\lambda)=K_{0}^{m} C_{g}(\lambda)$ is considered, where $K_{0}^{m}$ is a simultaneously stabilizing controller of order $m$ designed by HIFOO [3]. The results of both cases are then summarized in Table IV.

Theorem 2 initialized by the parameter-dependent state feedback controller $K_{s f}(\lambda)$ of Theorem 4 results in the following reduced-order $H_{\infty}$ controllers:

$$
\begin{aligned}
K^{m=0} & =\left[\begin{array}{ccc}
1.9407 & 12.3911
\end{array}\right] \\
K^{m=1} & =\left[\begin{array}{c|cc}
-4.3979 & 0 & 0 \\
\hline 0 & 2.2321 & 14.3543
\end{array}\right]
\end{aligned}
$$

For the second case, the following HIFOO controllers are used to initialize $K_{s f}(\lambda)$ :

$$
\begin{aligned}
K_{0}^{m=0} & =\left[\begin{array}{c|cc}
0.0958 & 0.7670
\end{array}\right] \\
K_{0}^{m=1} & =\left[\begin{array}{c|cc}
-1.1754 & 0.2729 & -1.8780 \\
\hline 0.6998 & 0.3876 & 0.24434
\end{array}\right]
\end{aligned}
$$

Theorem 2 initialized by $K_{s f}(\lambda)$ obtained by the above 
initial controllers leads to the following $H_{\infty}$ controllers:

$$
\begin{aligned}
& K^{m=0}=\left[\begin{array}{lll}
0.0965 & 0.8012
\end{array}\right] \\
& K^{m=1}=\left[\begin{array}{c|cc}
-1.8044 & 0.1048 & -3.1589 \\
\hline 0.9039 & 0.3921 & 3.1998
\end{array}\right]
\end{aligned}
$$

As mentioned in [18], the proposed approach of [34] leads to the lowest $H_{\infty}$ upper bound 37.20 for $m=0,1$ and the full-order control design method in [35] does not find any feasible solution.

\section{CONCLUSions}

In this paper, the problem of fixed-order $H_{\infty}$ controller design of LTI systems subject to polytopic uncertainty is considered. To this end, necessary and sufficient conditions based on the concept of strictly positive realness (SPRness) of a special transfer function are developed. The proposed conditions depend on a parameter-dependent gain determined through a parameter-dependent state feedback controller. The robust stability and robust $H_{\infty}$ performance of the closedloop polytopic systems are guaranteed via homogeneous polynomially parameter-dependent Lyapunov matrices. Simulation examples show the efficiency of the proposed fixedorder $H_{\infty}$ controller design approach.

\section{REFERENCES}

[1] M. Fu and Z.-Q. Luo, "Computational complexity of a problem arising in fixed order output feedback design," Systems and Control Letters, vol. 30, no. 5, pp. 209-215, 1997.

[2] P. Apkarian and D. Noll, "Nonsmooth $H_{\infty}$ synthesis," IEEE Trans. on Automatic Control, vol. 51, no. 1, pp. 71-86, 2006.

[3] S. Gumussoy and M. L. Overton, "Fixed-order $H_{\infty}$ controller design via HIFOO, a specialized nonsmooth optimization package," in American Control Conference, Seattle, USA, 2008, pp. 2750-2754.

[4] J. V. Burke, D. Henrion, and A. S. L. M. L. Overton, "HIFOO: A MATLAB package for fixed-order controller design and $H_{\infty}$ optimization," in Fifth IFAC Symposium on Robust Control Design, Toulouse, 2006.

[5] T. Iwasaki, "The dual iteration for fixed-order control," IEEE Trans. on Automatic Control, vol. 44, no. 4, pp. 783-788, April 1999.

[6] D. Peaucelle and D. Arzelier, "An efficient numerical solution for $\mathrm{H}_{2}$ static output feedback synthesis," in European Control Conference (ECC), Porto, Portugal, 2001.

[7] A. Karimi and G. Galdos, "Fixed-order $H_{\infty}$ controller design for nonparametric models by convex optimization," Automatica, vol. 46, no. 8, pp. 1388-1394, 2010.

[8] S. Wang and J. H. Chow, "Low-order controller design for SISO systems using coprime factors and LMI," IEEE Trans. on Automatic Control, vol. 45, no. 6, pp. 1166-1169, June 2000.

[9] D. Henrion, M. Sebek, and V. Kucera, "Positive polynomials and robust stabilization with fixed-order controllers," IEEE Trans. on Automatic Control, vol. 48, no. 7, pp. 1178-1186, 2003.

[10] F. Yang, M. Gani, and D. Henrion, "Fixed-order robust $H_{\infty}$ controller design with regional pole assignment," IEEE Trans. on Automatic Control, vol. 52, no. 10, pp. 1959-1963, 2007.

[11] A. Karimi, H. Khatibi, and R. Longchamp, "Robust control of polytopic systems by convex optimization," Automatica, vol. 43, no. 6, pp. 1395-1402, 2007.

[12] C. A. R. Crusius and A. Trofino, "Sufficient LMI conditions for output feedback control problems," IEEE Trans. on Automatic Control, vol. 44, no. 5, pp. 1053-1057, 1999.

[13] D. Mehdi, E. K. Boukas, and O. Bachelier, "Static output feedback design for uncertain linear discrete time systems," IMA Journal of mathematical control and information, vol. 21, pp. 1-13, 2004.

[14] K. H. Lee, J. H. Lee, and W. H. Kwon, "Sufficient LMI conditions for $H_{\infty}$ output feedback stabilization of linear discrete-time systems," IEEE Trans. on Automatic Control, vol. 51, no. 4, pp. 675-680, 2006.
[15] X. Du and G.-H. Yang, "LMI conditions for $H_{\infty}$ static output feedback control of discrete-time systems," in 47th IEEE Conference on Decision and Control, Cancun, Mexico, 2008, pp. 5450-5455.

[16] H. R. Moreira, R. C. L. F. Oliveira, and P. L. D. Peres, "Robust $\mathrm{H}_{2}$ static output feedback design starting from a parameter-dependent state feedback controller for time-invariant discrete-time polytopic systems," Optimal Control Applications and Methods, vol. 32, pp. 113, 2011.

[17] K. Dabboussi and J. Zrida, "Sufficient dilated LMI conditions for $H_{\infty}$ static output feedback robust stabilization of linear continuous-time systems," Journal of Applied Mathematics, vol. 2012, pp. 1-13, 2012.

[18] C. M. Agulhari, R. C. L. F. Oliveira, and P. L. D. Peres, "LMI relaxations for reduced-order robust $H_{\infty}$ control of continuous-time uncertain linear systems," IEEE Trans. on Automatic Control, vol. 57, no. 6, pp. 1532-1537, 2012.

[19] J. Dong and G.-H. Yang, "Robust static output feedback control synthesis for linear continuous systems with polytopic uncertainties," Automatica, vol. 49, pp. 1821-1829, 2013.

[20] M. S. Sadabadi and A. Karimi, "Fixed-order $H_{\infty}$ and $H_{2}$ controller design for continuous-time polytopic systems: An LMI-based approach," in European Control Conference (ECC), Zurich, Switzerland, 2013, pp. 1132-1137.

[21] — "An LMI formulation of fixed-order $H_{\infty}$ and $H_{2}$ controller design for discrete-time systems with polytopic uncertainty," in 52nd IEEE Conference on Decision and Control, Florence, Italy, 2013, pp. 2453-2458.

[22] A. Karimi and M. S. Sadabadi, "Fixed-order controller design for state space polytopic systems by convex optimization," in 5th IFAC Symposium on System Structure and Control, Grenoble, France, 2013, pp. 683-688.

[23] M. S. Sadabadi and A. Karimi, "Fixed-order controller design of linear systems," Technical Report, Automatic Control Laboratory, Ecole Polytechnique Fédérale de Lausanne (EPFL), 2014.

[24] S. Boyd, L. E. Ghaoui, E. Feron, and V. Balakrishnan, Linear Matrix Inequalities in System and Control Theory. Philadelphia: SIAM, 1994.

[25] J. C. Ghaoui, F. Oustry, and M. Ait-Rami, "A cone complementarity linearisation algorithm for static output feedback and related problems," IEEE Trans. on Automatic Control, vol. 42, no. 8, pp. 11711176, 1997.

[26] R. C. L. F. Oliveira and P. L. D. Peres, "Parameter-dependent LMIs in robust analysis: Characterisation of homogeneous polynomially parameter-dependent solutions via LMI relaxations," IEEE Trans. on Automatic Control, vol. 52, no. 7, pp. 1334-1340, 2007.

[27] C. M. Agulhari, R. C. L. F. de Oliveira, and P. L. D. Peres, "Robust LMI Parser: A computational package to construct LMI conditions for uncertain systems," in XIX Brazilian Conference on Automation (CBA 2012), Campina Grande, PB, Brazil, 2012, pp. 2298-2305.

[28] J. Löfberg, "YALMIP: A toolbox for modeling and optimization in MATLAB," in CACSD Conference, 2004. [Online]. Available: http://control.ee.ethz.ch/ joloef/yalmip.php

[29] J. F. Sturm, "Using SeDuMi 1.02, A Matlab toolbox for optimization over symmetric cones," Optimization Methods and Software, vol. 11, pp. 625-653, 1999.

[30] J. Dong and G.-H. Yang, "Static output feedback control synthesis for linear systems with time-invariant parametric uncertainties," IEEE Trans. on Automatic Control, vol. 52, no. 10, pp. 1930-1936, 2007.

[31] D. Arzelier, E. N. Gryazina, D. Peaucelle, and B. T. Polyak, "Mixed LMI/randomized methods for static output feedback control design," in American Control Conference, Baltimore, MD, USA, 2010, pp. 46834688.

[32] U. Shaked, "An LPD approach to robust $H_{2}$ and $H_{\infty}$ static outputfeedback design," IEEE Trans. on Automatic Control, vol. 48, no. 5, pp. 866-872, 2003.

[33] M. S. Sadabadi and A. Karimi, "Robust performance analysis of single parameter-dependent systems with polynomially parameter-dependent Lyapunov matrices," in 19th IFAC World Congress (IFAC WC 2014), Cape Town, South Africa, 2014, pp. 6141-6146.

[34] I. Yaesh and U. Shaked, "Robust reduced-order output-feedback $H_{\infty}$ control," in 6th IFAC Sym. Robust Control Design, Haifa, Israel, 2009, pp. $155-160$

[35] J. C. Geromel, R. H. Korogui, and J. Bernussou, " $H_{2}$ and $H_{\infty}$ robust output feedback control for continuous time polytopic systems," IET Control Theory and Applications, vol. 1, no. 5, pp. 1541-1549, September 2007. 